\title{
Light modulators and deflectors based on polariton effects
}

\section{Skettrup, Torben}

Published in:

Journal of Applied Physics

Link to article, DOI:

10.1063/1.328530

Publication date:

1981

Document Version

Publisher's PDF, also known as Version of record

Link back to DTU Orbit

Citation (APA):

Skettrup, T. (1981). Light modulators and deflectors based on polariton effects. Journal of Applied Physics, 52(10), 6292-6295. https://doi.org/10.1063/1.328530

\section{General rights}

Copyright and moral rights for the publications made accessible in the public portal are retained by the authors and/or other copyright owners and it is a condition of accessing publications that users recognise and abide by the legal requirements associated with these rights.

- Users may download and print one copy of any publication from the public portal for the purpose of private study or research.

- You may not further distribute the material or use it for any profit-making activity or commercial gain

- You may freely distribute the URL identifying the publication in the public portal

If you believe that this document breaches copyright please contact us providing details, and we will remove access to the work immediately and investigate your claim. 


\title{
Light modulators and deflectors based on polariton effects
}

\author{
T. Skettrup \\ Physics Laboratory III, Technical University of Denmark, DK-2800 Lyngby, Denmark
}

(Received 16 October 1980; accepted for publication 17 March 1981)

\begin{abstract}
The possibility of constructing light modulators and deflectors based on polariton effects is considered. The polariton is a mixed complex consisting of a superposition of a one-photon state and a crystal excitation state. By influencing the crystal excitation state by means of external fields, the polariton, and hence the photon part, can be deflected or modulated. The connection with geometrical optics is established, and it is shown that the deflection is due to a gradient in the refraction index created by the applied external field. Several examples with electric, magnetic, and stress fields, and temperature gradients are shown with orders of magnitude of deflections computed.
\end{abstract}

PACS numbers: $78.20 . J q, 78.20 . L s, 78.20 . \mathrm{Nv}$

\section{INTRODUCTION}

There is a strong need for a simple device that can deflect or modulate light. The present work is concerned with the possibility of constructing such a device utilizing the polariton properties of light propagating through a solid in a spectral region close to a resonance. It turns out that in this spectral region, light and matter become so mixed that it is possible to deflect or modulate the light by influencing the matter properties by means of external fields.

\section{POLARITONS}

The polariton was introduced in 1958 by Hopfield ${ }^{\prime}$ as the quantum of the light propagating through a solid, i.e., the quantum of the coupled electromagnetic field and the polarization field of the solid. The relative magnitudes of these fields are related by the dielectric constant at the angular frequency $\omega$ of the light

$$
\epsilon(\omega)=\epsilon^{\prime}+Q /\left(\omega_{0}^{2}-\omega^{2}\right),
$$

where $\omega_{0}$ is the resonance frequency for the phenomenon giving rise to the polarization (i.e., TO-phonons, excitons, etc.), $Q$ is a parameter accounting for the strength of the interaction between electromagnetic waves and polarization waves, and $\epsilon^{\prime}$ is the "background dielectric constant" observed if $Q=0$. The relationship between the angular frequency $\omega$ and the wave vector $k$ of the polariton is obtained from the usual condition for light propagation:

$$
k^{2}=\left(\omega^{2} / c_{0}^{2}\right) \epsilon(\omega),
$$

where $c_{0}$ is the speed of light in a vacuum. The wave function for the polariton was also derived by Hopfield.' A simpler form, derived from a microscopic interaction model by Skettrup, ${ }^{2}$ is

$$
\psi=\left(a_{1} \psi_{0} \phi_{1}+i a_{2} \psi_{1} \phi_{0}\right) e^{\cdots i \omega t},
$$

where $\omega$ is given by Eq. (2), $\psi_{0}$ and $\phi_{0}$ are ground states of crystal and vacuum, respectively, while $\psi_{1}$ and $\phi_{1}$ are the excited crystal state (i.e., exciton state) and the one-photon state with wave vector k. Further,

$$
\begin{aligned}
& a_{1}=\left(\omega_{0}-\omega\right) /\left[\left(\omega_{0}-\omega\right)^{2}+\omega_{c}^{2}\right], \\
& a_{2}=\omega_{c} /\left[\left(\omega_{0}-\omega\right)^{2}+\omega_{c}^{2}\right],
\end{aligned}
$$

where

$$
\omega_{\mathrm{c}}=\left(Q / 4 \epsilon^{\prime}\right)^{1 / 2} .
$$

Hence, the polariton is a stationary state that can be considered as a linear superposition of a one-photon state and a crystal excitation (in the following considered as an exciton ${ }^{3}$. At the frequencies

$$
\omega_{s}=\omega_{0} \pm \omega_{c} \text {, }
$$

the photon and exciton content in the polariton is equal. For

$$
\omega_{0}-\omega_{c}<\omega<\omega_{0}+\omega_{c},
$$

the exciton content is greater than the photon content, i.e., the polariton is matterlike. At resonance the photon part vanishes and close to resonance the polariton is essentially an exciton. Hence in the frequency range [Eq. (8)] the quanta of the light propagating through the solid (the polaritons) are more like matter than like light, but they travel with the speed of light of the solid given by Eq. (2). Then we encounter the unusual situation that "a piece of matter" travels with the speed of light. However, as seen from Eq. (2) the dispersion relation is far from being masslike and the velocity is far from the light velocity in vacuum.

When the propagating "light" in the spectral range [Eq. (8)] possesses matter properties, there exists the possibility of influencing these matter properties by external fields in order to modulate or deflect the propagating beam. In Table I some II-VI compounds with pronounced polariton effects are shown. They can alloy together and their resonance frequencies can, therefore, cover most of the visible region. It is seen that the spectral region (8) for these materials ranges from $10-25 \mathrm{~nm}$. Within these spectral ranges modulators based on polaritons can, in principle, be constructed. In the following we examine more closely $\mathrm{ZnO}$, the II-VI compound with most pronounced polariton effects. At $80 \mathrm{~K}$, $\hbar \omega_{0}=3.42 \mathrm{eV}(363 \mathrm{~nm})$ for the $C$-exciton in $\mathrm{ZnO}$. Hence, a polariton modulator can work in the spectral range $350 \mathrm{~nm}<\lambda<376 \mathrm{~nm}$ for $\mathrm{ZnO}$ at $80 \mathrm{~K}$. However, the crystal is absorbing strongly in most of this spectral range. The absorption coefficient follows the so-called Urbach's rule for many of these materials, ${ }^{4}$

$$
\alpha=\alpha_{0} \exp \left[-\sigma\left(E_{0}-\hbar \omega\right) / k T\right],
$$

where $\alpha_{0}, \sigma$, and $E_{0}$ are material parameters, and $k T$ is the thermal energy. From the absorption curves ${ }^{5}$ for $\mathrm{ZnO}$ the smallest absorption found in the above mentioned range is 
TABLE I. Exciton resonance energies and wavelengths $\hbar \omega_{0}$ and $\lambda_{0}$ at $80 \mathrm{~K}$ for different II-VI compounds. The spectral ranges of matter-like polaritons $\left(2 \hbar \omega_{c}\right.$ and $\left.\Delta \lambda\right)$ are computed from Eq. (6) by means of parameters obtained from Refs. 6 and 7

\begin{tabular}{lllllll}
\hline \hline & $\begin{array}{l}\hbar \omega_{0} \\
(\mathrm{eV})\end{array}$ & $\begin{array}{l}\lambda_{0} \\
(\mathrm{~nm})\end{array}$ & $\begin{array}{l}\hbar^{2} Q \\
(\mathrm{eV})^{2}\end{array}$ & $\epsilon^{\prime}$ & $\begin{array}{l}2 \hbar \omega_{c} \\
(\mathrm{meV})\end{array}$ & $\begin{array}{l}\Delta \lambda \\
(\mathrm{nm})\end{array}$ \\
\hline $\mathrm{ZnS}$ & 3.85 & 322 & 0.12 & 8.3 & 120 & 10 \\
$\mathrm{ZnO}$ & 3.42 & 363 & 0.37 & 6.2 & 244 & 25 \\
$\mathrm{Cds}$ & 2.55 & 486 & 0.070 & 8.0 & 94 & 18 \\
$\mathrm{CdSe}$ & 1.82 & 681 & 0.022 & 7.7 & 54 & 20 \\
\hline \hline
\end{tabular}

about $10 \mathrm{~cm}^{-1}$ at $375 \mathrm{~nm}$. Increasing the temperature to room temperature (polariton effects are expected to be present also at high temperatures ${ }^{2}$ ) implies a shift of resonance energy to $\hbar \omega_{0}=3.335 \mathrm{eV}(370 \mathrm{~nm}) .{ }^{6}$ The absorption at 3.22 $\mathrm{eV}\left[\hbar\left(\omega_{0}-\omega_{c}\right)\right]$ is then according to Ref. (5) about 100 $\mathrm{cm}^{-1}$. At $80 \mathrm{~K}$ then the crystal thickness should be of the order $1 \mathrm{~mm}$ and at room temperature about $1 / 10 \mathrm{~mm}$ in order to have Eq. (8) fulfilled and obtain a reasonable transmission. However, the thickness can be chosen larger if the frequency goes beyond $\omega_{s}$ in Eq. (7). This means that the photon contribution exceeds the exciton contribution in the polariton wave function [Eq. (3)]. Still, it is, of course, possible to influence the polariton by influencing the exciton properties, but the effect is less pronounced as indicated by Eq. (10) below.

\section{POLARITON MODULATOR}

We wish to compute the order of magnitude of deflection of a polariton, the exciton part of which is acted on by the constant force, $F_{0}$. According to Eq. (3) the average force on the polariton is then

$$
F=a_{2}^{2} F_{0}
$$

where $a_{2}$ [Eq. (5)] is the fraction of excitonic wave function content present in the polariton state. Hence, the force depends on the interaction strength $Q$ and gradually vanishes as $\left(\omega_{0}-\omega\right)$ increases. Using the geometry shown in Fig. 1 where the force is directed along the $y$ direction and the incoming polariton propagates in the $x$ direction, we obtain from the equation of motion in $k$ space,

$$
\hbar \frac{d k_{y}}{d t}=F
$$

for change in the polariton wave vector. Assuming $F$ constant,

$$
k_{y}=\frac{F}{\hbar}\left(\frac{d}{v_{g}}\right)
$$

where $d$ is the thickness of the crystal and $v_{g}$ is the polariton group velocity. This gives rise to a deflection angle

$$
\alpha=\frac{k_{y}}{k_{x}}=\frac{F d}{\hbar \omega} \frac{v_{p}}{v_{g}},
$$

where Eq. (2) was used and $v_{p}$ is the phase velocity of the polariton. From Eq. (2) we obtain

$$
\begin{aligned}
v_{p} / v_{g} & =1+\left[4 \omega_{c}^{2} \omega^{2} /\left(\omega_{\mathrm{ex}}^{2}-\omega^{2}\right)\left(\omega_{L}^{2}-\omega^{2}\right)\right] \\
& \simeq 1+\left[\omega_{c}^{2} /\left(\omega_{0}-\omega\right)\left(\omega_{L}-\omega\right)\right],
\end{aligned}
$$

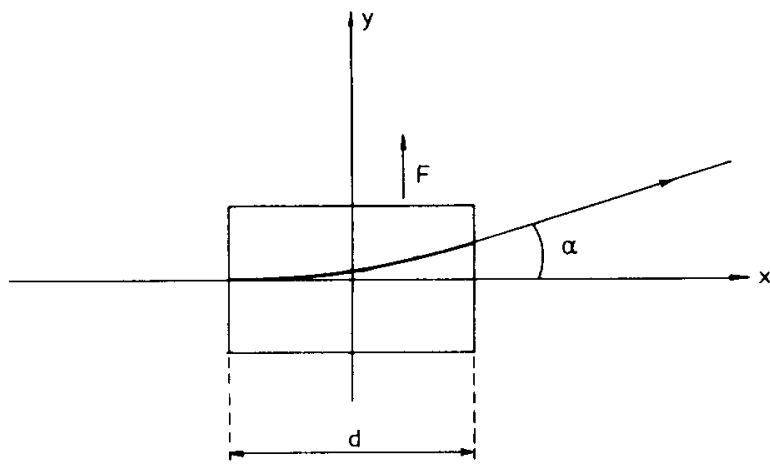

FIG. 1. Deflection of polariton propagating in the $x$ direction.

where

$$
\omega_{L}^{2}=\omega_{0}^{2}+4 \omega_{c}^{2} .
$$

For $\left(\omega_{0}-\omega\right) \sim \omega_{c}$, Eq. (14) yields $v_{p} / v_{g} \sim 2$. This factor approximately cancels the $a_{2}^{2}$ factor of Eq. (10). This is assumed to be the case in the estimates given in Sec. V.

\section{CONNECTION WITH GEOMETRICAL OPTICS}

In order to establish the connection with the more conventional description in terms of refractive indices, we wish to derive Eq. (13) in the framework of geometrical optics. The differential equation for rays ${ }^{8}$ can in the geometry of Fig. 1 be written as

$$
n \frac{d^{2} y}{d x^{2}}=\frac{d n}{d y}
$$

where $n$, the refractive index, is the square root of $\epsilon$, given in Eq. (1).

Since the force $F_{0}$ acting on the exciton is given by

$$
F_{0}=-\left[\frac{d\left(\hbar \omega_{0}\right)}{d y}\right]
$$

the refractive index becomes dependent on $y$ [see Eq. (1)]. Hence,

$$
\frac{d n}{d y}=\frac{d n}{d \omega_{0}} \frac{d \omega_{0}}{d y}=-\frac{d n}{d \omega}\left(-\frac{F_{0}}{\hbar}\right)=\frac{c}{\hbar \omega}\left(\frac{1}{v_{g}}-\frac{1}{v_{p}}\right) F_{0}
$$

using Eqs. (1) and (2). Equation (18) shows that $d n / d y$ is constant. Then the light ray follows a circular path as seen from Eq. (16), and the deflection angle $\alpha$ is given by

$$
\alpha=d \frac{d^{2} y}{d x^{2}}=\frac{d}{n} \frac{d n}{d y}=\frac{d}{\hbar \omega}\left(\frac{v_{p}}{v_{g}}-1\right) F_{0}=\frac{F d}{\hbar \omega} \frac{v_{p}}{v_{g}}
$$

Q.E.D., since

$$
F / F_{0}=1-\frac{v_{g}}{v_{p}}=\frac{4 \omega_{c}^{2} \omega^{2}}{\left(\omega_{0}^{2}-\omega^{2}\right)^{2}+4 \omega_{c}^{2} \omega_{c}^{2}} \simeq \frac{\omega_{c}^{2}}{\left(\omega_{0}-\omega\right)^{2}+\omega_{c}^{2}}
$$

which is the same result as in Eq. (10).

Hence, in the language of geometrical optics the optical path length in the $x$ direction is varying as a function of $y$. This causes a deflection (in analogy with, for example, a 
prism). In the language of polaritons, however, the physics behind is more clearly displayed, i.e., the deflection occurs because the exciton (which is travelling with the speed of light of the crystal and carrying the energy of the incoming photon to the exit surface) is deflected due to an applied force, $F_{0}$.

\section{EXAMPLES}

We shall in the following use Eq. (13) for computing actual orders of magnitude for different kinds of forces. The pure exciton consists of an electron and a hole bound together by their coulombic interaction. It is thus a neutral particle which can not be deflected by homogeneous electric or magnetic fields. (Homogeneous electric fields can, of course, imply absorption modulation due to the Franz-Keldysh effect.) Since the exciton possesses a magnetic dipole moment of the order of one Bohr magneton $\mu_{B}$, there exists a possibility of deflecting the polariton in a inhomogeneous magnetic field. The force is

$$
F_{y}=\mu_{B} \frac{d B}{d y} .
$$

Using a value of $d B / d y=100 \mathrm{Vs} / \mathrm{m}^{3}$, we find from Eq. (13) in the case of $\mathrm{ZnO}$.

$$
\alpha / d=1.7 \mu \mathrm{rad} / \mathrm{mm} \text {. }
$$

This deflection is, therefore, too small to be of any practical importance.

In order to deflect the exciton by other inhomogeneous fields, the exciton resonance energy must be dependent on the field considered. As an example we consider a thermal gradient along the $y$ direction. The exciton is then acted on by the constant force

$$
F_{y}=-\frac{d\left(\hbar \omega_{0}\right)}{d y}=-\frac{d\left(\hbar \omega_{0}\right)}{d T} \frac{d T}{d y} .
$$

Assuming a gradient of $50 \mathrm{~K} / \mathrm{mm}$ and using $d\left(\hbar \omega_{0}\right) / d T=-0.36 \mathrm{meV} / \mathrm{K}$ for $\mathrm{ZnO},{ }^{6} \mathrm{Eq}$. (13) yields

$$
\alpha / d=5.3 \mathrm{mrad} / \mathrm{mm} \text {. }
$$

This order of magnitude is reasonable and this thermal device can be of practical importance.

An inhomogeneous stress field also implies an inhomogeneous bandgap and hence a force acting on the exciton. Consider as an example an inhomogeneous uniaxial stress $S$ along the $y$ direction. The force on the exciton is then

$$
F_{y}=-\frac{d\left(\hbar \omega_{0}\right)}{d y}=-\frac{d\left(\hbar \omega_{0}\right)}{d S} \frac{d S}{d y} .
$$

From Langer et al. ${ }^{9} d\left(\hbar \omega_{0}\right) / d S \simeq 0.9 \mathrm{meV} / \mathrm{kbar}$ is obtained for the $C$ exciton in $\mathrm{ZnO}$. Assuming $d S / d y \simeq 1 \mathrm{kbar} / \mathrm{mm}$ we find from Eq. (13)

$$
\alpha / d=-0.26 \mathrm{mrad} / \mathrm{mm} \text {. }
$$

This effect is then about 20 times less pronounced than the thermal effect in Eq. (24). However, it is faster and can, therefore, prove more useful.

An inhomogeneous electric field $E$ can also deflect the polariton via the quadratic Stark effect which shifts the exciton resonance energy. The force due to the Stark effect is given by ${ }^{10}$

$$
F_{y}=\frac{9}{2}\left(4 \pi \epsilon_{0} \epsilon a_{B}^{2}\right) E \frac{d E}{d y},
$$

where $a_{B}$ is the Bohr radius of the exciton and $\epsilon$ is the low frequency value of the dielectric constant. For $\mathrm{ZnO}$ we find with $\epsilon=7.9$ (Ref. 7) and $a_{B}=18 \AA($ Ref. 11)

$$
\alpha / d=4.2 \times 10^{-17} \frac{m^{2}}{V^{2}} E \frac{d E}{d y} .
$$

Hence, very strong and inhomogeneous electric fields are needed to obtain deflections of practical magnitudes. In order to have the same order of magnitude as for the thermal deflector [Eq. (24)], the field must be $E \sim 100 \mathrm{kV} / \mathrm{cm}$ with $d E / d y \sim 1 \mathrm{MV} / \mathrm{cm}^{2}$. Fields of this order of magnitude can be produced in connection with Schottky barriers. There exists then a possibility for constructing light deflector devices which work with applied electric fields. Such devices can be very fast. A disadvantage (from the point of view of deflection) is that the transmission will be modulated because the absorption coefficient depends on the electric field (FranzKeldysh effect).

Finally, we consider the important example of a charged exciton (a three-particle complex consisting of 2 electrons and one hole or vice versa). Although such a complex, to the author's knowledge, has not been reported, it is likely to exist since its molecular analogue, the ionized hydrogen molecule $\mathrm{H}_{2}^{+}$is known to be stable. The force acting on a charge exciton from an applied electric field $E$ is very large,

$$
F_{y}=e E_{y} \text {. }
$$

Applying only $1 \mathrm{~V} / \mathrm{cm}$ results in a deflection for the $\mathrm{ZnO}$ case,

$$
\alpha / d=30 \mathrm{mrad} / \mathrm{mm} \text {. }
$$

This order of magnitude of deflection for so small electric fields certainly can imply practical applications.

\section{CONCLUSION}

It has been shown that it is possible to construct light modulators or deflectors based on polariton effects. In order to obtain sufficiently strong deflectors the wavelength of the incoming light must not be too far from the excitonic resonance. The excitonic resonance can be varied through most of the visible spectrum by alloying different II-VI compounds. Orders of magnitude of deflection were estimated for different applied forces.

'J. J. Hopfield, Phys. Rev. 112, 1555 (1958).

${ }^{2}$ T. Skettrup Phys. Rev. B (to be published).

${ }^{3}$ R. S. Knox, Theory of Excitons (Academic, New York, 1965)

${ }^{4}$ M. V. Kurik, Phys. Status Solid A8, 9 (1971).

${ }^{5}$ G. Hvedstrup Jensen and T. Skettrup, Phys. Status Solid (B) 60, 169 (1973).

${ }^{6} \mathrm{G}$. Hvedstrup Jensen, Phys. Statis Solid (B) 64, K51 (1974).

${ }^{7}$ B. Segall, Phys. Rev. B 163, 769 (1976).

${ }^{8}$ M. Born and E. Wolf, Principles of Optics (Pergamon, London-Paris, 
1975), p. 122.

D. W. Langer, R. N. Euwema, K. Era, and T. Koda, Phys. Rev. B 2, 4005 (1970).
${ }^{10}$ L. D. Landau and E. M. Lifshitz, Quantum Mechanics (Pergamon, London-Paris, 1959)

${ }^{1}$ T. Skettrup and I. Baislev, Phys. Status Solid 40, 93 (1970). 